\title{
EXTRACCIÓN DE OLEORRESINA DE CÚRCUMA (Curcuma longa) UTILIZANDO ETANOL COMO SOLVENTE
}

\section{EXTRACTION OF OLEORESIN FROM TURMERIC (Curcuma longa) USING ETHANOL AS SOLVENT}

\author{
Sandra Lorena Blandón Navarro ${ }^{1 *}$ \\ Carlos Mario Ponce Arévalo ${ }^{2}$
}

(Recibido/received: 29-octubre-2021; aceptado/accepted: 30-noviembre-2021)

RESUMEN: Este estudio tuvo como objetivo evaluar el proceso de extracción de la oleorresina de cúrcuma utilizando etanol (97\% en volumen) como solvente. En este sentido, se llevaron a cabo experimentos de extracción de la oleorresina usando como materia prima la cúrcuma seca (13\% de humedad) con granulometría de $4.75 \mathrm{~mm}$. El tiempo de extracción fue de 24 horas a 40 ${ }^{\circ} \mathrm{C}$. Los resultados mostraron que el rendimiento de extracción de la oleorresina de $3.18 \pm 0.14 \%$ fue influenciado por la granulometría gruesa de la materia prima, dado que fue menor al reportado en estudios previos, donde se utilizó cúrcuma molida con granulometría menor a $500 \mu \mathrm{m}$. En relación a la cantidad de solución adherida al sólido residual de cúrcuma se determinó que fue de $1.51 \pm 0.20 \mathrm{~g}$ de solución/ $\mathrm{g}$ de sólidos. De los resultados se puede inferir que el uso de etanol en la extracción de oleorresina es técnicamente viable, sin embargo, su desempeño está influenciado por las características de la materia prima y el pretratamiento del material sólido anterior a la etapa de extracción (secado y reducción de tamaño).

PALABRAS CLAVE: Curcuma longa; secado; solvente alcohólico; extracción sólido-líquido

ABSTRACT: This study aimed to evaluate the turmeric oleoresin extraction process using ethanol ( $97 \%$ by volume) as solvent. In this sense, oleoresin extraction experiments were carried out using dry turmeric (moisture $13 \%$ ) with a particle size of $4.75 \mathrm{~mm}$ as raw material. The extraction time was 24 hours at $40^{\circ} \mathrm{C}$. The results showed that the oleoresin extraction yield of $3.18 \pm 0.14 \%$ was influenced by the coarse particle size of the raw material, since it was lower than that reported in previous studies, where ground turmeric with a particle size less than $500 \mu \mathrm{m}$ was used. In relation to the amount of solution adhered to the residual turmeric solid, it was determined that it was 1.51 $\pm 0.20 \mathrm{~g}$ of solution $/ \mathrm{g}$ of solids. From the results it can be inferred that the use of ethanol in the extraction of oleoresin is technically feasible, however, its performance is influenced by the characteristics of the raw material and the pretreatment of the solid material prior to the extraction stage (drying and reduction of size).

${ }^{1}$ Profesora titular, Universidad Nacional de Ingeniería, Sede Regional del Norte, Estelí, Nicaragua. *Autor para correspondencia, email: sandra.blandon@norte.uni.edu.ni

2 Ingeniero Agroindustrial. Inspector Auxiliar del Instituto de Protección y Sanidad Agropecuaria (IPSA), municipio de Condega, Estelí, Nicaragua. 
KEYWORDS: Curcuma longa; drying; alcoholic solvent; solid-liquid extraction

\section{INTRODUCCIÓN}

La cúrcuma (Curcuma longa) es una planta perteneciente a la familia de las Zingiberaceae. La planta alcanza la altura de un metro y se caracteriza por poseer un tallo corto y hojas copetudas. A su vez, los rizomas son cortos y gruesos y son la parte de la planta de la cual se prepara la especia (Gupta y Balasubrahmanyam, 1998; Joshi, Jain y Sharma, 2009).

La producción mundial de cúrcuma es de alrededor de 1,100,000 toneladas por año. Los principales exportadores son India (62.6\%), Vietnam (5.37\%), Myanmar (5.27\%), Indonesia $(2.48 \%)$ y China (2\%). Entre los importadores figuran Estados Unidos (13\%), India (11.3\%), Irán (5.1\%), Alemania (4.79\%) y Bangladesh (4.76\%) (Observatory of Economic Complexity, 2020).

En el 2019 Nicaragua exportó 92 toneladas de cúrcuma. Para ese año ocurrió un aumento en las exportaciones de esta especia de 3.37 \% en comparación con el año 2018. Entre 2017 y 2019, en Nicaragua las exportaciones de cúrcuma crecieron en $46.03 \%$ (Selina Wamucii, 2020), lo que evidencia que Nicaragua está teniendo mayor presencia en el mercado de las especias.

Cabe mencionar que la cúrcuma puede ser procesada para posteriormente ser usada como colorante en alimentos, cosméticos y textiles. En India se utiliza como ingrediente de formulaciones medicinales para enfermedades comunes (Garg, Bansal, Gupta y Kumar, 1999).

En relación a la composición química de la cúrcuma Gupta y Balasubrahmanyam (1998) reportan un contenido de $13.1 \%$ de humedad, $6.3 \%$ de proteína, $5.1 \%$ de grasa, $69.4 \%$ de carbohidratos, $3.5 \%$ de minerales, $2.6 \%$ de fibras y carotenos calculados como vitamina A de $50 \mathrm{lU} / 100 \mathrm{~g}$.

Además, la cúrcuma puede presentar alrededor de $3.5 \%$ de aceites esenciales, 2.5-6.0 \% de curcuminoides y $5.7 \%$ de oleorresinas (Aniesrani Delfiya, Thangavel, Natarajan, Kasthuri y Kailappan, 2015). Según Aniesrani Delfiya et al. (2015) la oleorresina de cúrcuma es un colorante natural que se obtiene del rizoma molido por extracción con solvente (acetona, dicloruro de etileno o etanol). Además, está compuesta por pigmentos curcuminoides (30-55 \%) y aceite volátil (15$25 \%$ ) (Aniesrani Delfiya et al., 2015).

Los curcuminoides se refieren a un grupo de compuestos fenólicos, los cuales están químicamente relacionados. La curcumina, es el principal polifenol curcuminoide encontrado en la cúrcuma y es el responsable de las propiedades medicinales y farmacológicas de la misma (González-Albadalejo et al., 2015). En la industria de alimentos, la curcumina se agrega a alimentos como grasas, sopas, repostería, productos cárnicos y bebidas, para impartir color. También se utiliza como antioxidante para prevenir la rancidez (Sogi, Sharma, Oberoi y Wani, 2010).

Tomando en cuenta los aspectos mencionados, la presente investigación tuvo como finalidad evaluar el uso de etanol a $40^{\circ} \mathrm{C}$ en el proceso de extracción de oleorresina de cúrcuma, visualizando este producto como alternativa de valor agregado para esta especia. 


\section{MATERIALES Y MÉTODOS}

Los experimentos de extracción de la oleorresina de cúrcuma fueron realizados en el Laboratorio de Agroindustria de la Universidad Nacional de Ingeniería, sede Regional del Norte (UNI Norte, Estelí, Nicaragua).

Este estudio es una investigación experimental que constó de las etapas de acondicionamiento de la cúrcuma (secado) y la extracción de la oleorresina. Rodríguez Moguel (2005) plantea que los estudios experimentales son investigaciones prospectivas, donde hay manipulación de una variable experimental en condiciones controladas, con el fin de describir de qué modo o por qué causa se produce la situación o acontecimiento particular.

\section{Muestra}

Los rizomas de cúrcuma, peso de $5 \mathrm{~kg}$, fueron comprados directamente al productor, comunidad Santa Josefina, municipio de Matagalpa, departamento de Matagalpa, Nicaragua. Posteriormente, pasaron por una operación de inspección, para asegurar que los rizomas presentaran una textura firme y sólida y estuvieran libres de daños.

\section{Métodos analíticos}

La determinación del contenido de humedad de los rizomas se hizo a través del método AOAC 925.45 (A.O.A.C., 2005), utilizando el secador (Thermo Fisher Scientific, modelo PR305225G) a una temperatura de $60^{\circ} \mathrm{C}$ por 24 horas. Este análisis se realizó por quintuplicado.

\section{Acondicionamiento de muestra para la extracción de oleorresina}

Las principales operaciones a las que fue sometida la cúrcuma en la etapa de acondicionamiento de la materia prima, se describen a seguir.

\section{a. Lavado}

Consistió en lavar los rizomas con agua potable y un cepillo de fibras suaves, delgado y pequeño que permitiera eliminar la tierra acumulada en la cúrcuma.

\section{b. Desinfección}

Esta etapa es de gran importancia en la calidad, seguridad y vida pos cosecha del rizoma. El proceso consistió en sanear la superficie del rizoma, a través del uso de un desinfectante químico (Hipoclorito de sodio). Para lograr este saneamiento del rizoma, se sumergió en una solución de agua y desinfectante en una concentración de 25 ppm.

\section{c. Oreado}

Para eliminar el agua superficial de los rizomas, estos se dejaron en reposo separados unos de otros sobre la mesa durante 24 horas.

\section{d. Reducción de tamaño}


La reducción de tamaño se realizó con un rallador de cocina para obtener tiras delgadas (fajas) que permitieran una mejor extracción y drenaje del extracto.

\section{e. Secado de las tiras de cúrcuma}

Debido a la influencia del contenido de humedad del producto en el rendimiento de extracción, las tiras de cúrcuma se sometieron a secado, misma que se realizó en el secador (Thermo Fisher Scientific, modelo PR305225G) durante 12 horas a temperaturas $50 \pm 1^{\circ} \mathrm{C}$, llevando el producto hasta una humedad final de aproximadamente $13 \%$.

\section{Experimentos de extracción de la oleorresina}

El solvente utilizado para la extracción de oleorresina fue alcohol etílico al $97 \%$ en volumen. Se añadió en una proporción 1:5 a la ralladura de cúrcuma, donde por cada gramo de ralladura de cúrcuma se agregaron 5 gramos de alcohol etílico al $97 \%$.

Se seleccionó la proporción 1:5 considerando que en esta se logra una imbibición completa del sólido en el solvente. Con proporciones 1:3 y 1:4 un porcentaje de la muestra siempre quedaba fuera de contacto con el solvente. Además, Ferreira (2016) en la evaluación del uso de etanol para la extracción sólido-líquido de aceite de soja mostró que utilizando la proporción sólido: solvente de 1:1.5 después de la extracción quedaba una cantidad considerable de aceite retenido en el sólido, mientras que en proporciones mayores, como 1:4 y 1:6 se extrae mayor cantidad de aceite, quedando una menor cantidad de aceite retenido en la fase sólida.

En la etapa de extracción se utilizó Erlenmeyer de $100 \mathrm{~mL}$ en el cual se colocó el sólido (previamente pesado), seguidamente se adicionó el solvente y se realizó agitación (5 minutos) para distribuirlo en toda la matriz y facilitar la transferencia de masa durante la extracción. A seguir, se colocó un tapón en la boca del Erlenmeyer y se dejó en reposo durante 24 horas a 40 ${ }^{\circ} \mathrm{C}$.

\section{Filtración del extracto}

Esta operación se realizó para separar las partículas sólidas y extracto, y así obtener una solución libre de sólidos, para ello se utilizó colador con diámetro de orificios de la pantalla de $1 \mathrm{~mm}$.

\section{Separación de la oleorresina del solvente}

Esta etapa se realizó colocando el extracto en placas Petri y sometiéndolas a secado a $50{ }^{\circ} \mathrm{C}$ durante 8 horas para eliminar el solvente (etanol). El producto que quedó en las placas se pesó y se registró el valor para calcular el rendimiento. 


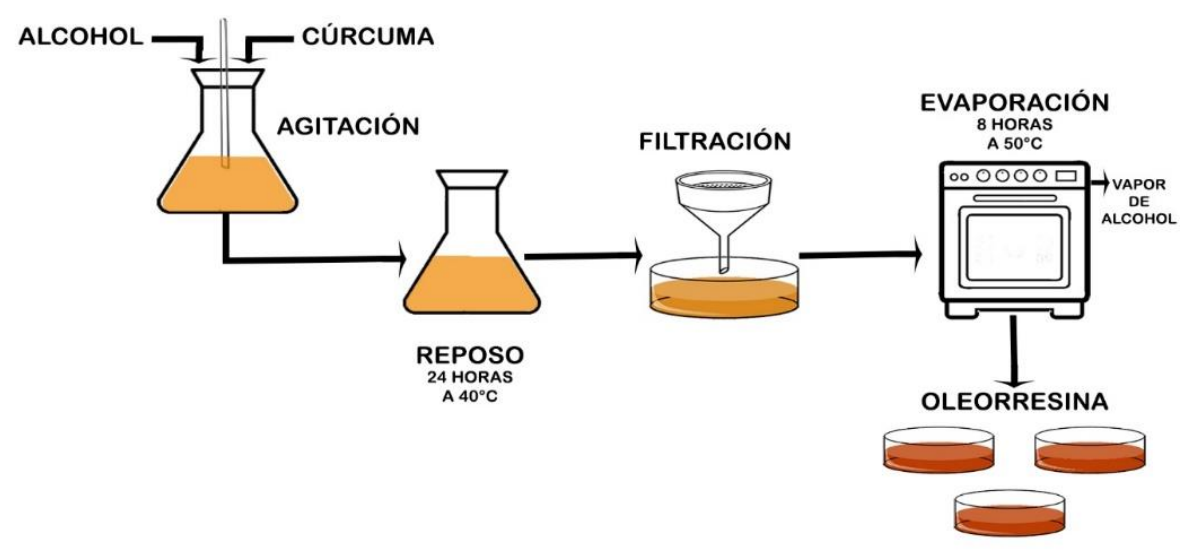

Figura 1. Extracción de la oleorresina de cúrcuma

El rendimiento de extracción de la oleorresina consistió en el pesaje de la materia prima inicial (cúrcuma seca) y la cantidad de oleorresina obtenida al final del proceso. En la ecuación 1, se muestra la fórmula utilizada.

$$
\% \text { Rendimiento }=\frac{g \text { final de oleorresina }}{g \text { Inicial de cúrcuma seca }} \times 100 \quad \text { Ecuación } 1
$$

Estos experimentos fueron realizados por quintuplicado.

\section{Cantidad de solución en el sólido residual}

Después de la separación del extracto de la fase sólida mediante filtración el sólido se dejó escurrir por 5 minutos y, posteriormente, se pesó. A continuación, el sólido se colocó en placas Petri para eliminar el solvente y humedad mediante secado por 24 horas. La cantidad en gramos de esta solución adherida por gramo de sólido residual se calculó de la diferencia entre el peso del sólido antes del secado y después del secado.

Procesamiento y Análisis de los resultados

Los datos de la investigación se procesaron en el Software estadístico InfoStat @ versión 2018 (Di Rienzo et al., 2018) para el cálculo de medias y desviación estándar.

\section{RESULTADOS Y DISCUSIÓN}

La cúrcuma se recepcionó, se inspeccionó, fue lavada y oreada para ser utilizada en los experimentos. En la Figura 2 se presentan las imágenes de algunos de los rizomas utilizados. 


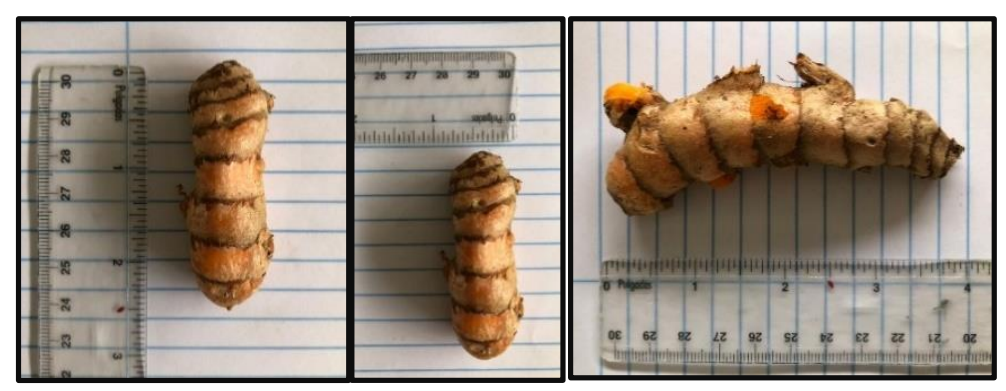

Figura 2. Rizomas de cúrcuma (Curcuma longa)

\section{Determinación del contenido de humedad}

Se determinó que la cúrcuma contiene un $86.2 \pm 0.49 \%$ de humedad. Este valor está dentro del rango de humedad de 62 a 86 \% reportado por Garg et al. (1999) para rizomas de 27 accesiones recolectadas de la región subhimalaya de Terai de la India. Un mayor contenido de humedad fue encontrado por Hirun, Utama-ang y Roach (2014) que determinaron para la cúrcuma fresca 91.52 $\%$ de humedad. Hirun et al. (2014) sugieren que la diferencia en el contenido de humedad podría deberse a la temporada de cosecha y las variedades de cúrcuma.

\section{Acondicionamiento de la cúrcuma}

Los rizomas lavados y oreados fueron llevados al rallado en tiras. Después, las tiras fueron sometidas a secado, como fue descrito en la metodología. En la siguiente figura se muestra la cúrcuma antes y después del secado.

a)

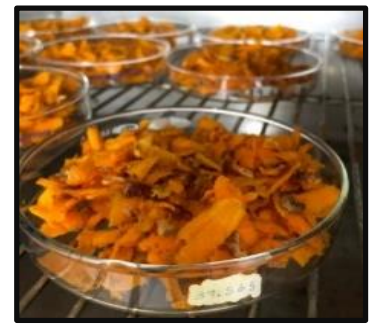

b)

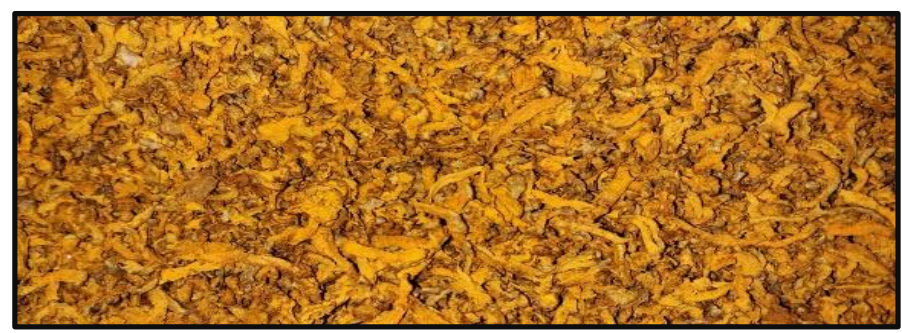

Figura 3. a) Cúrcuma en tiras antes del secado b) Cúrcuma seca

Después del secado se observó encogimiento de las tiras de cúrcuma y una leve disminución en la intensidad del color al compararlas con las tiras de cúrcuma antes del secado. Según Sagar y Suresh Kumar (2010), durante el secado convencional por convección se producen varios cambios en las propiedades físicas de los tejidos vegetales, como la pérdida de color y el cambio de textura, así como los cambios químicos que afectan el sabor, el contenido de nutrientes y la contracción. Lewicki y Duszczyk (1998) sugieren que la contracción y los cambios de estructura causados por el secado determinan la capacidad del material de absorber agua y de hincharse en una etapa posterior de hidratación. No obstante, la pérdida de calidad del producto vegetal puede ser menor al llevar a cabo el proceso de secado a temperaturas más bajas (Sagar y Suresh Kumar, 2010). De este modo, es posible inferir que en el caso de realizar el secado de la cúrcuma a temperaturas mayores a $60^{\circ} \mathrm{C}$ pueden conducir a mayor degradación de los curcuminoides. 
Por tamizado, se determinó que la mayoría de las partículas presentó un tamaño de $4.75 \mathrm{~mm}$. En la tabla 1 se presenta el tamaño determinado para la cúrcuma seca rallada. Este material fue el utilizado en la extracción de la oleorresina.

Tabla 1. Tamaño de las partículas de la Cúrcuma seca rallada

\begin{tabular}{ccc}
\hline $\mathbf{N}^{\circ}$ de Tamiz & Medida & \% de Masa Retenida \\
\hline$\geq 4$ & $+4.75 \mathrm{~mm}$ & 16.78 \\
4 & $4.75 \mathrm{~mm}$ & 51.95 \\
10 & $2.00 \mathrm{~mm}$ & 27.72 \\
20 & $850 \mu \mathrm{m}$ & 3.54 \\
\hline
\end{tabular}

Rendimiento de extracción de la oleorresina

Después de realizadas las etapas que conllevó el proceso de extracción se determinó que utilizando etanol al $97 \%$ como solvente se obtienen rendimientos de $3.18 \pm 0.14 \%$ de oleorresina. Este producto presentaba un aspecto oleoso y fácil de untar, en ella está contenida la curcumina, uno de los principales componentes de la oleorresina de cúrcuma.

El rendimiento de extracción de oleorresina del presente estudio es menor al rango reportado por Chempakam y Parthasarathy (2008) de $7.9 \%$ a $10.4 \%$. También difiere de los rendimientos obtenidos por Souza y Glória (1998) en extractor Soxhlet por un período de contacto de 4 horas, usando hexano como solvente y posteriormente etanol o acetona, obteniendo rendimientos de oleorresina de 8 a $10 \%$. Souza y Glória (1998) usaron cúrcuma molida con $10 \%$ de humedad y granulometría de $250 \mu \mathrm{m}$. Chien et al. (1990) señalan que el rendimiento de la extracción de sustancias oleosas disminuye significativamente al aumentar el tamaño de las partículas. Los autores sugieren que, en partículas finas, el soluto está más expuesto en su superficie, lo que facilita la extracción. Por lo tanto, el bajo rendimiento determinado en este trabajo puede ser debido a que la cúrcuma utilizada presentaba una granulometría gruesa. Kurmudle, Bankar, Bajaj, Bule y Singhal (2011) mencionan que el tamaño de partícula menor de $500 \mu \mathrm{m}$ es deseable para una máxima eficiencia de extracción de la oleorresina de cúrcuma.

Otras razones a las que se le puede atribuir el bajo rendimiento obtenido en esta investigación son las condiciones de separación del solvente de la oleorresina por evaporación, ya que la fracción de los volátiles presentes en la oleorresina (de 15-25 \% reportado por Aniesrani Delfiya et al., 2015) puede haberse evaporado durante esta etapa.

Cantidad de solución adherida al sólido residual

Se determinó que la cantidad de solución adherida al sólido residual de cúrcuma fue de $1.51 \pm$ $0.20 \mathrm{~g}$ de solución/g de sólidos. Navarro y Rodrigues (2018) sugieren que las características del sólido, contenido de humedad y operaciones previas de acondicionamiento inciden sobre la cantidad de solución que queda adherida al sólido residual después de la extracción. Ferreira (2016) en sus experimentos de extracción de aceite de soja con etanol encontró mayor cantidad de solución adherida en soja húmeda expandida en comparación con soja seca expandida. Estudio de Ruiz Saldívar (2020) encontró que en la pulpa de nancite (Byrsonima crassifolia) la 
cantidad de solución retenida fue de $0.55 \pm 0.02 \mathrm{~g}$ de solución/ $\mathrm{g}$ de sólidos. En dicho estudio el solvente utilizado fue etanol en grado absoluto (99.9 \% de pureza) y pulpa de nancite seca. Por lo que en la presente investigación es posible inferir que el contenido de humedad de la cúrcuma utilizada en la extracción (13\% después del secado parcial), su composición química, el grado de hidratación del etanol utilizado (97\%) y la granulometría del material incidieron en este nivel de solución retenida.

La determinación de la cantidad de solución adherida en el sólido residual es relevante, dado que entre mayor sea la cantidad de solución retenida, mayor será la cantidad de energía necesaria para separar el solvente del sólido residual.

\section{CONCLUSIONES}

Al realizar el proceso de extracción de oleorresina de cúrcuma a partir de rizomas en tiras con tamaños de $4.75 \mathrm{~mm}$, contenido de humedad de $13 \%$ y utilizando etanol $97 \%$ como solvente se obtienen rendimientos de $3.18 \pm 0.14 \%$ de oleorresina. Después de la extracción, la cantidad de solución adherida al sólido residual de cúrcuma fue de $1.51 \pm 0.20 \mathrm{~g}$ de solución/ $\mathrm{g}$ de sólidos. Este valor es mayor al reportado en estudios con otras materias primas y puede ser atribuido a las características del sólido utilizado, composición química y operaciones previas de acondicionamiento.

De este modo, es posible inferir que la extracción de oleorresina de cúrcuma utilizando etanol como solvente es técnicamente viable. No obstante, el rendimiento de extracción puede aumentar al utilizar cúrcuma molida con granulometría de $500 \mu \mathrm{m}$.

\section{AGRADECIMIENTOS}

Los autores agradecen la colaboración del Ing. Jandir David Gutiérrez Martínez e Ing. Christopher Octavio Olivas Rodríguez durante el desarrollo de los experimentos en el laboratorio de Agroindustria de la Universidad Nacional de Ingeniería, sede Regional del Norte.

\section{REFERENCIAS}

A.O.A.C. (2005). Official Methods of Analysis of AOAC International (18va ed.). Arlington, Virginia: AOAC International.

Aniesrani Delfiya, D. S., Thangavel, K., Natarajan, N., Kasthuri, R. y Kailappan, R. (2015). Microencapsulation of Turmeric Oleoresin by Spray Drying and In Vitro Release Studies of Microcapsules. Journal of Food Process Engineering, 38(1), 37-48. https://doi.org/10.1111/jpe. 12124

Chempakam, B. y Parthasarathy, V. A. (2008). Turmeric. En: V. A. Parthasarathy, B. Chempakam y T. J. Zachariah (Eds.), Chemistry of spices. Cabi. (pp. 97-118). Londres, Reino Unido: 
CABI.

Chien, J. T., Hoff, J. E., Lee, M. J., Lin, H. M., Chen, Y. J., \& Chen, L. F. (1990). Oil extraction of dried ground corn with ethanol. The Chemical Engineering Journal, 43(3), B103-B113. https://doi.org/10.1016/0300-9467(90)80017-7

Di Rienzo, J. A., Casanoves, F., Balzarini, M. G., Gonzalez, L., Tablada, M., \& Robledo, C. W. (2018). Infostat versión 2018. Córdoba, Argentina: Universidad Nacional de Córdoba. Recuperado de: http://www.infostat.com.ar

Ferreira, M. C. (2016). Evaluación del uso de etanol para la extracción sólido-líquido de aceite de Soja para la producción de biodiesel etílico mediante análisis de equilibrio liquido-liquido. Tesis para optar al título de Doutora em Engenharia de Alimentos. Campinas, Brasil: Universidade Estadual de Campinas - Faculdade de Engenharia De Alimentos.

Garg, S. N., Bansal, R. P., Gupta, M. M. y Kumar, S. (1999). Variation in the rhizome essential oil and curcumin contents and oil quality in the land races of turmeric Curcuma longa of North Indian plains. Flavour and Fragrance Journal, 14(5), 315-318. https://doi.org/10.1002/(SICI)1099-1026(199909/10)14:5<315::AID-FFJ838>3.0.CO;2-U

González-Albadalejo, J., Sanz, D., Claramunt, R. M., Lavandera, J. L., Alkorta, I. y Elguero, J. (2015). Curcumin and curcuminoids: chemistry, structural studies and biological properties. Real Academia Nacional de Farmacia, 81, 278-310.

Gupta, R. K. y Balasubrahmanyam, L. (1998). The turmeric effect. World Patent Information, 20, 185-191.

Hirun, S., Utama-ang, N. y Roach, P. D. (2014). Turmeric (Curcuma longa L.) drying: an optimization approach using microwave-vacuum drying. Journal of Food Science and Technology, 51(9), 2127-2133. https://doi.org/10.1007/s13197-012-0709-9

Joshi, P., Jain, S. y Sharma, V. (2009). Turmeric (Curcuma longa) a natural source of edible yellow colour. International Journal of Food Science \& Technology, 44(12), 2402-2406. https://doi.org/10.1111/j.1365-2621.2009.01914.x

Kurmudle, N. N., Bankar, S. B., Bajaj, I. B., Bule, M. V. y Singhal, R. S. (2011). Enzyme-assisted three phase partitioning: A novel approach for extraction of turmeric oleoresin. Process Biochemistry, 46(1), 423-426. https://doi.org/10.1016/j.procbio.2010.09.010

Lewicki, P. P. y Duszczyk, E. (1998). Color change of selected vegetables during convective air drying. International Journal of Food Properties, 1(3), 263-273. https://doi.org/10.1080/10942919809524582

Navarro, S. L. B. y Rodrigues, C. E. C. (2018). Macadamia Oil Extraction With Alcoholic Solvents: Yield and Composition of Macadamia Oil and Production of Protein Concentrates From Defatted Meal. European Journal of Lipid Science and Technology, 120(7). https://doi.org/10.1002/ejlt.201800092

Observatory of Economic Complexity. (2020). Turmeric (curcuma). Fecha de acceso: Noviembre 11, 2021. Recuperado de: https://oec.world/en/profile/hs92/turmeric-curcuma 
Rodríguez Moguel, E. A. (2005). La Investigación. In E. A. Rodríguez Moguel (Ed.), Metodología de la Investigación (Primera ed, p. 25). Tabasco, México: Universidad Juárez Autónoma de Tabasco.

Ruiz Saldívar, D. M. (2020). Evaluación de la extracción de aceite de fruto de nancite (Byrsonima Crassifolia L. Hbk) con solventes alcohólicos y caracterización de su efecto antimicrobiano. Programa de Maestría en Procesamiento de Alimentos. Facultad de Ingenería Química. Universidad Nacional de Ingeniería.

Sagar, V. R. y Suresh Kumar, P. (2010). Recent advances in drying and dehydration of fruits and vegetables: a review. Journal of Food Science and Technology, 47(1), 15-26. https://doi.org/10.1007/s13197-010-0010-8

Selina Wamucii. (2020). Global Food and Agriculture Marketplace. Nicaragua Turmeric Market Insights. Fecha de acceso: Noviembre 18, 2021. Recuperado de: https://www.selinawamucii.com/insights/market/nicaragua/turmeric/

Sogi, D. S., Sharma, S., Oberoi, D. P. S. y Wani, I. A. (2010). Effect of extraction parameters on curcumin yield from turmeric. Journal of Food Science and Technology, 47(3), 300-304. https://doi.org/10.1007/s13197-010-0047-8

Souza, C. R. A. y Glória, M. B. A. (1998). Chemical analysis of turmeric from Minas Gerais, Brazil and comparison of methods for flavour free oleoresin. Brazilian Archives of Biology and Technology, 41(2), 218-224. https://doi.org/10.1590/S1516-89131998000200008

\section{SEMBLANZA DE LOS AUTORES}

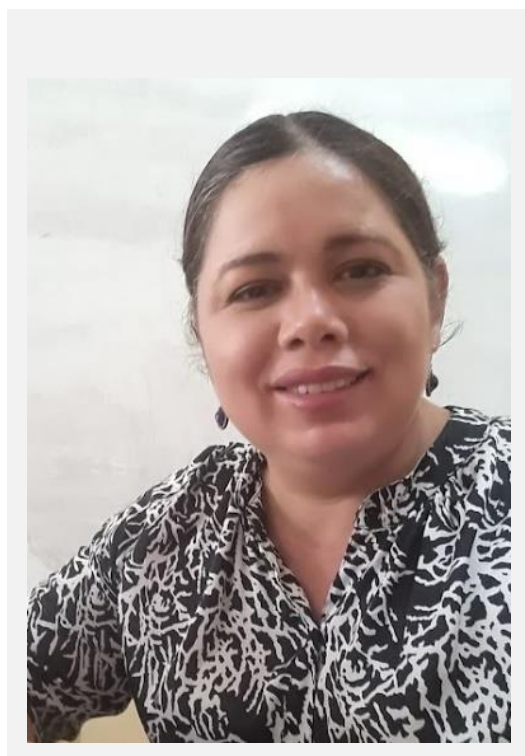

Sandra Lorena Blandón Navarro: Es Doctora en Ciencias de Ingeniería de Alimentos, Universidad de Sao Paulo (USP), Brasil (2016), con maestría en Procesamiento de Alimentos, Universidad Nacional de Ingeniería (UNI), Nicaragua (2009) y graduada de Ingeniería Química en la misma universidad (2003). Actualmente es Profesora titular de la UNI a nivel de grado y posgrado, en la carrera de Ingeniería agroindustrial y en la maestría en Procesamiento de Alimentos de la Facultad de Ingeniería Química (FIQ-UNI). Posee diplomados de Modelo Educativo Institucional (IPN-México), Educación Online (UOLUNI, Managua, Nicaragua) y Estrategias de enseñanza y aprendizaje aplicadas a los estudios de alimentos (ISEKI FOOD-4 PROJECT, Atenas, Grecia). Además, posee experiencia en el área de Ciencia y Tecnología de Alimentos, con énfasis en desarrollo de productos, aprovechamiento de residuos de la agroindustria e ingeniería de separaciones. 


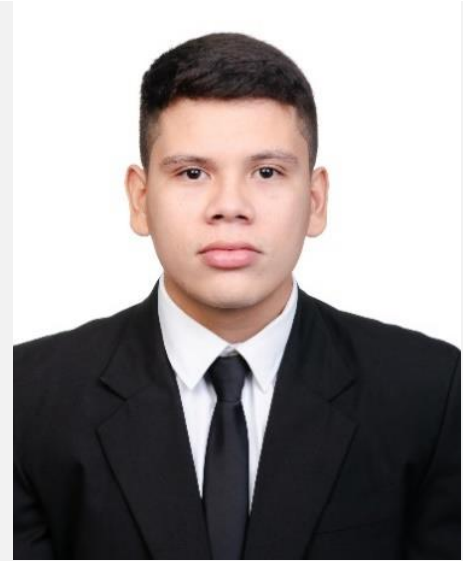

Carlos Mario Ponce Arévalo: Es graduado de la carrera de Ingeniería Agroindustrial - Universidad Nacional de ingeniería (2021). Ha realizado prácticas profesionales en los rubros del tabaco, panificación y granos básicos. Actualmente se desempeña como Inspector Auxiliar del Instituto de Protección y Sanidad Agropecuaria (IPSA) en la empresa productora de carne bovina Nica Beef Packers S.A (Condega, Estelí, Nicaragua). Posee conocimientos sobre procesos agroindustriales, control de calidad e inocuidad alimentaria. 